\title{
La agricultura tradicional como legado agroecológico para la humanidad
}

Miguel A. Altieri | Dpto. of Environmental Science, Policy, \& Management, University of California (Berkeley)

URL de la contribución <www.iaph.es/revistaph/index.php/revistaph/article/view/4960>

\section{RESUMEN}

Los sistemas agrícolas complejos, diversos y localmente adaptados desarrollados por milenios por agricultores a lo largo del mundo constituyen un patrimonio agrícola que abarca unos 10 millones de hectáreas, las cuales además de proveer de alimentos a la humanidad proveen una combinación vital de servicios sociales, culturales, ecológicos y económicos.

Estos sistemas han contribuido no solamente con extraordinarios paisajes de belleza estética, sino que también proveen alimentos a una porción significativa de la población, mientras mantienen la biodiversidad agrícola base para manejar agroecosistemas resilientes y constituyen un patrimonio cultural valioso de importancia mundial.

Muchos de los nuevos modelos de agricultura que la humanidad urgentemente necesita en una época de cambio global, para transicionar hacia formas de agricultura que sean más ecológicas, biodiversas, locales, sostenibles y socialmente justas deberán estar arraigadas en la racionalidad ecológica de la agricultura tradicional que representa ejemplos establecidos de formas acertadas de agricultura local.

\section{Palabras clave}

Agricultura tradicional | Agroecología | Patrimonio cultural | Servicios ecológicos | 


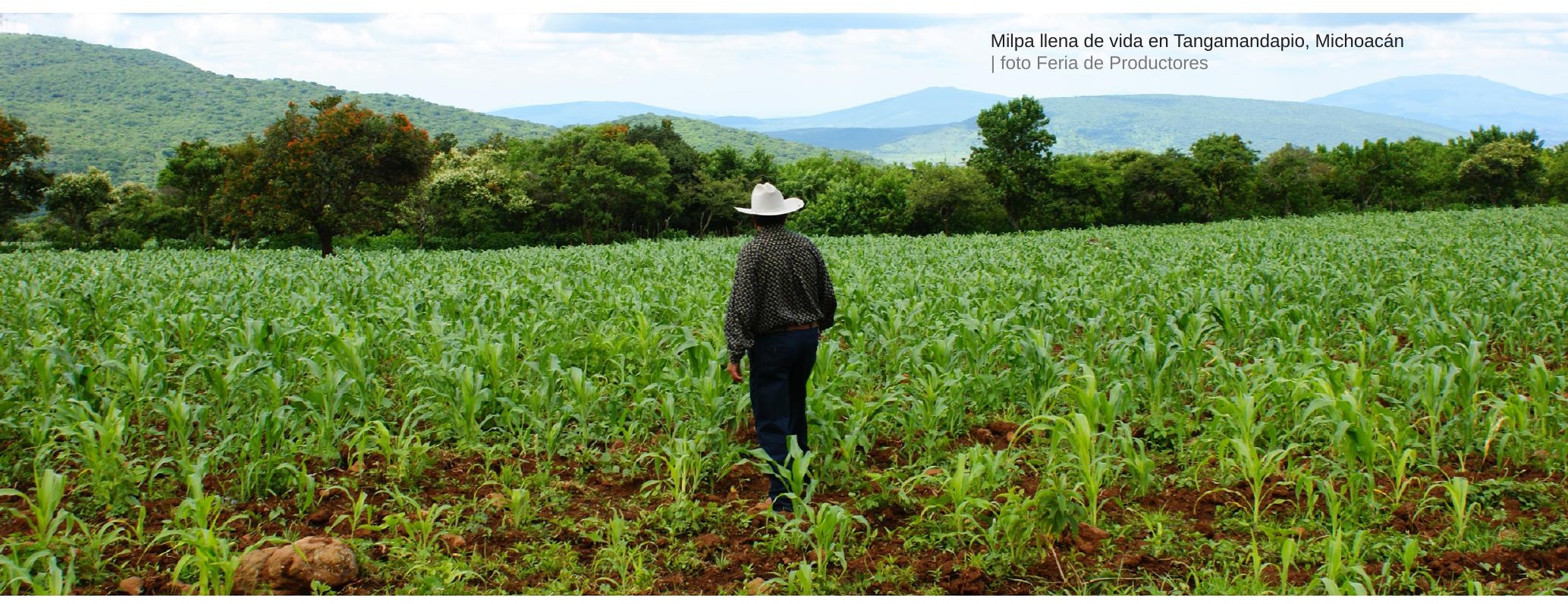

\section{Traditional agriculture: an agroecological legacy for humanity}

\section{ABSTRACT}

Complex, diverse and locally adapted agricultural systems developed for millennia by farmers throughout the world constitute an agricultural heritage that covers some 10 million hectares, which in addition to providing food to humanity provide a vital combination of social and cultural, ecological and economic services.

These systems have contributed not only with extraordinary landscapes of aesthetic beauty, but also provide food to a significant portion of the population, while maintaining the agricultural biodiversity key to manage resilient agroecosystems and constitute a valuable cultural heritage of global importance.

Many of the new models of agriculture that humanity urgently needs in a time of global change, to transition towards forms of agriculture that are more ecological, biodiverse, local, sustainable and socially just will have to be rooted in the ecological rationale of traditional agriculture that represents established examples of successful forms of local agriculture.

\section{Key words}

Traditional agriculture | Agroecology | Agricultural Heritage | Ecosystem services |

Cómo citar: Altieri, M.A. (2021) La agricultura tradicional como legado agroecológico para la humanidad. Revista PH, n. ${ }^{\circ}$ 104, 2021, pp. 180-197 <www.iaph. es/revistaph/index.php/revistaph/article/view/4960> DOI 10.33349/2021.104.4960

Enviado: 02/06/2021 | Aceptado: 19/07/2021 | Publicado: 01/10/2021 


\section{INTRODUCCIÓN}

Durante milenios, comunidades de agricultores tradicionales han desarrollado sistemas agrícolas complejos, diversos y localmente adaptados. Estos sistemas han sido manejados con combinaciones ingeniosas de técnicas y prácticas de eficacia comprobada, que usualmente han conducido a la seguridad alimentaria de las comunidades rurales y a la conservación de los recursos naturales y la biodiversidad. Este conjunto de agroecosistemas dispersos a lo largo del mundo constituyen un patrimonio agrícola que abarca unos 10 millones de hectáreas, los cuales además de proveer de alimentos a la humanidad proveen una combinación vital de servicios sociales, culturales, ecológicos y económicos. Estos sistemas han contribuido no solamente con extraordinarios paisajes de belleza estética, sino también en el mantenimiento de la biodiversidad agrícola, agroecosistemas resilientes y un patrimonio cultural valioso de importancia mundial. Por encima de todo, estos sistemas proveen múltiples bienes y servicios, seguridad alimentaria y los medios de subsistencia para millones de pobres y pequeños campesinos (Koohafkan y Altieri 2017).

Los sistemas agrícolas tradicionales se han ido conformando durante siglos, a partir de una co-evolución cultural y biológica y representan la experiencia acumulada de los campesinos en su interacción con el medioambiente, sin contar ni con insumos externos, ni con capitales, ni con el denominado saber científico. Haciendo uso de una autonomía ingeniosa, de un saber vivencial y de unos recursos cercanos, los campesinos han creado sistemas agrícolas sobre la base de una diversidad de cultivos, de árboles y de animales en el espacio y en el tiempo, lo que les ha permitido maximizar la seguridad de las cosechas en medios marginales y variables y con un espacio y unos recursos limitados (Wilken 1987). Estos sistemas se han desarrollado partiendo de un conocimiento basado no solo en la observación, sino también en el aprendizaje experimental. Este enfoque es obvio en la selección y obtención de variedades de semillas locales y en la experimentación con nuevos métodos de cultivo para superar determinados obstáculos bióticos y abióticos. La mayoría de los agricultores tradicionales poseen un conocimiento íntimo de sus alrededores, especialmente dentro de un radio geográfico y cultural próximo (DeWalt 1994).

La permanencia de millones de hectáreas agrícolas bajo el antiguo manejo tradicional en la forma de campos elevados, terrazas, policultivos, sistemas agroforestales, etc., documenta una estrategia agrícola indígena exitosa y constituye un tributo a la creatividad e ingenio de los agricultores tradicionales. Este microcosmos de agricultura tradicional ofrece modelos prometedores ya que promueve la biodiversidad, prospera sin agroquímicos y sostiene producciones todo el año (Denevan 1995). De seguro, muchos de los nuevos modelos de agricultura que la humanidad necesita para transicionar 


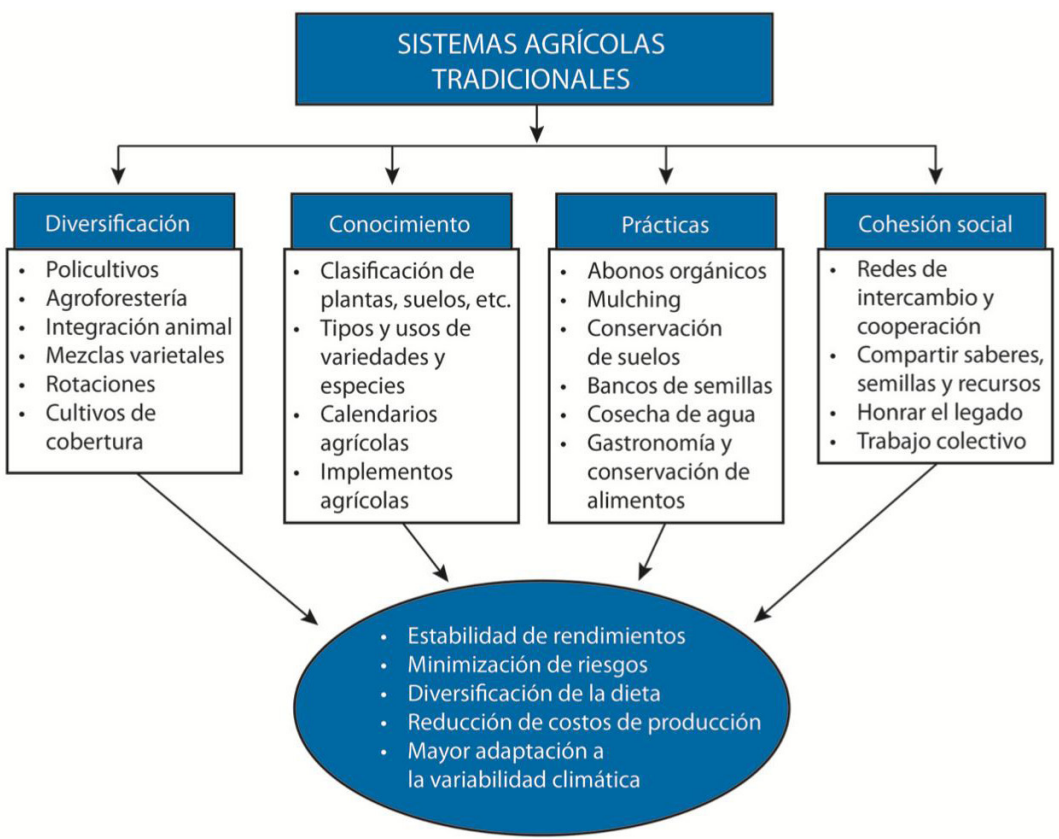

hacia formas de agricultura que sean más ecológicas, biodiversas, locales, sostenibles y socialmente justas deberán estar arraigadas en la racionalidad ecológica de la agricultura tradicional que representa ejemplos establecidos de formas acertadas de agricultura local. Tales sistemas han alimentado la mayor parte del mundo durante siglos y siguen alimentando a millones de personas en muchas partes del planeta (Altieri 2004).

A pesar de la inmensa variedad de sistemas agrícolas tradicionales, y de sus particularidades históricas y geográficas, la mayoría de los agroecosistemas tradicionales comparte, con gran parecido, los siguientes rasgos y características (Koohafkan y Altieri 2010):

$>$ Niveles muy altos de biodiversidad. Esta desempeña un papel en la regulación del funcionamiento del ecosistema y en la obtención de servicios con relevancia local y global.

> Sistemas ingeniosos de conservación de gestión de recursos edáficos e hídricos a nivel paisajístico, que mejoran la eficiencia de los agro-ecosistemas.

> Sistemas agrícolas diversificados que ofrecen una gran variedad de productos para la soberanía alimentaria local y nacional y la seguridad de los medios de vida.
Características socio-ecológicas de los sistemas agrícolas tradicionales que confieren resiliencia estabilidad productiva y soberanía alimentaria 
Sistema agroforestal rustico de café en Antioquia (Colombia) | foto M. A. Altieri

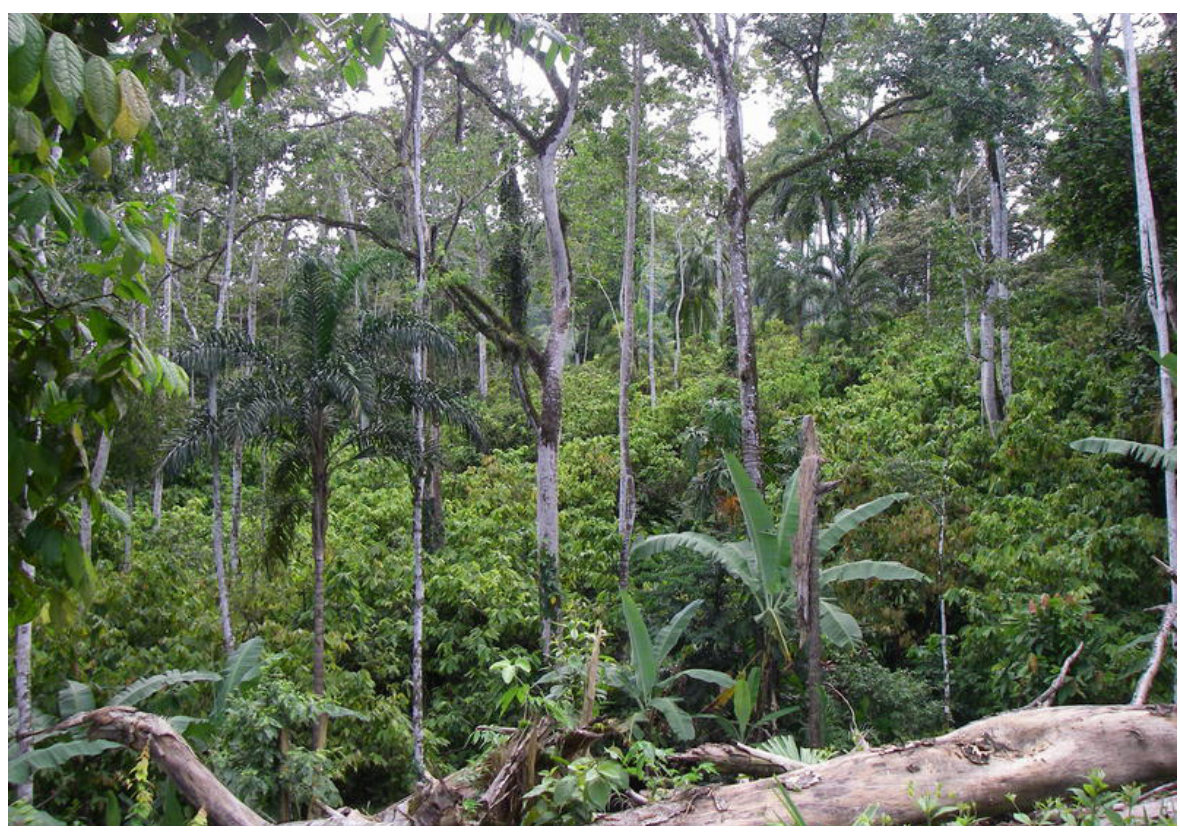

> Agroecosistemas que poseen una resistencia y una robustez para minimizar los riesgos ante la variabilidad y la estocasticidad.

$>$ Agroecosistemas alimentados por sistemas de conocimiento tradicionales con muchas innovaciones y tecnologías campesinas.

$>$ Valores culturales fuertes y formas de organización social colectivas, como instituciones consuetudinarias para la gestión agroecológica, acuerdos normativos para el acceso a los recursos y el reparto de beneficios, sistemas de valores, rituales, etc.

Dado que estos sistemas han resultado en el mantenimiento y la adaptación de paisajes excepcionales que incluye sistemas de conocimiento y biodiversidad agrícola de importancia mundial, en 2002, la FAO inició una iniciativa internacional para la gestión adaptativa de los sistemas de patrimonio agrícola de importancia mundial: Sistemas Importantes del Patrimonio Agrícola Mundial. SIPAM tiene como objetivo sentar las bases para el reconocimiento internacional, la conservación dinámica y el manejo sostenible de sistemas tradicionales, que incluyen el conocimiento etnoecológico que permite conservar, manejar y utilizar los agropaisajes y su biodiversidad agrícola. Comprender los principios y mecanismos que gobiernan la resiliencia y sostenibilidad de los SIPAM podría proveer las bases para el diseño de nuevos sistemas agrícolas capaces de producir alimentos en forma sostenible y amigable con la naturaleza (Koohafkan y Dela Cruz 2011). 


\section{LA AGRICULTURA TRADICIONAL Y CAMPESINA: EXTENSIÓN Y SIGNIFICANCIA}

Informes producidos por organizaciones como ETC Group y Grain estiman que unos 1500 millones de campesinos cultivan la tierra en alrededor de 380 millones de pequeñas fincas (menores de dos hectáreas de tamaño medio), En América Latina, las unidades de producción campesinas alcanzaron cerca de 16 millones a finales de los años ochenta, cifra que supone la ocupación de cerca de 60,5 millones de hectáreas, el 34,5\% del total la tierra cultivada. La población campesina incluye a 75 millones de personas que representan casi dos tercios de la población rural total de América Latina (Ortega 1986). Se estima que estas cifras se mantienen en la actualidad. El tamaño medio de finca de estas unidades se acerca a 1,8 hectáreas. Sin embargo, la contribución de la agricultura campesina al suministro de alimentos general en la región resulta significativa. En los años ochenta, aproximadamente el $41 \%$ de la producción agrícola destinada para consumo doméstico provino de la agricultura campesina, la cual produjo a nivel regional el $51 \%$ del maíz, el $77 \%$ de los frijoles y el $61 \%$ de las papas. Solo en Brasil, hay cerca de 4,8 millones de familias de agricultores familiares (alrededor del $85 \%$ del total de agricultores) que ocupan el $30 \%$ del total de la tierra agrícola del país. Dichas familias controlan cerca del $33 \%$ del área sembrada en maíz, el $61 \%$ en frijoles y el $64 \%$ de aquellas tierras cultivadas en yuca, lo que equivale al $84 \%$ de la yuca total y el $67 \%$ de todas las frijoles para el consumo nacional. En Ecuador, el sector campesino ocupa más del $50 \%$ del área dedicada a los cultivos alimenticios tales como maíz, frijoles, cebada y papa. En México, los campesinos ocupan, por lo menos, el $70 \%$ del área destinada al maíz y el 60 \% del área en frijoles. En Cuba, las campesinas y campesinos producen casi dos tercios de los alimentos del país en apenas un tercio de las tierras arables (Altieri y Toledo 2011).

Además del sector campesino y de los agricultores familiares, hay cerca de 50 millones de indigenas que pertenecen a unos 700 grupos étnicos, quienes viven y utilizan las regiones tropicales húmedas de la región. Cerca de 2 millones de ellos viven en el Amazonas y en México meridional. En México, la mitad de las zonas tropicales húmedas se utilizan por las comunidades indígenas y los "ejidos", en los cuales se desarrollan sistemas integrados de agricultura-silvicultura con la producción dirigida hacia la subsistencia y mercados a nivel local y regional (Toledo et ál. 1985).

África tiene aproximadamente 33 millones de fincas pequeñas, cantidad que representa el $80 \%$ de todas las fincas en la región. La mayoría de los agricultores africanos (muchos de ellos, mujeres) tienen el perfil de pequeños productores que controlan dos tercios de todas las fincas con área inferior a 2 hectáreas y el $90 \%$ de fincas menores a 10 hectáreas. La mayoría de los pequeños agricultores practican una agricultura de bajos-insumos, 
basada sobre todo en el uso de recursos locales, prácticamente sin utilización o poco uso de fertilizantes y semillas mejoradas. Esta agricultura de bajos-insumos produce la mayoría del grano; casi todas las raíces, cosechas de tubérculos y plátanos y la mayoría de legumbres (Richards 1985). Sin embargo, esta situación ha cambiado en las últimas dos décadas en tanto que la producción de alimento per cápita ha disminuido en África. Alguna vez autosuficientes en cereales, África ahora tiene que importar millones de toneladas para llenar el vacío. A pesar del incremento en las importaciones, los pequeños agricultores siguen produciendo la mayor parte del alimento de África (Richards 1985).

En Asia, solamente China posee casi la mitad de las pequeñas fincas de la región (193 millones de hectáreas), seguidas por la India con $23 \%$ y, detrás, Indonesia, Bangladesh y Vietnam. La mayoría de los más de 200 millones de agricultores que vive en Asia posee fincas con menos de 2 hectáreas de arroz. Tan solo en China hay probablemente 75 millones de agricultores de arroz, quienes aún practican métodos similares a los utilizados hace más de mil años. La mayoría del arroz producido por los pequeños agricultores asiáticos se produce en condiciones de pendiente, en terrazas inundadas o en laderas dependientes de lluvias (Hanks 1992).

Varios estudios han demostrado que los policultivos de arroz, por ejemplo arroz (Oryza sativa) con azolla (Azolla sp., ej. Azolla pinnata) o el arroz con peces (Oreochromis niloticus y Cyprinus carpio) y el arroz con patos (Anas platyrhynchos javanicus), reducen los niveles de uso de agroquímicos mientras se obtienen rendimientos de arroz similares a los de los sistemas de monocultivo convencionales. La presencia del helecho/alga Azolla en la superficie del agua de los campos de arroz pueden reducir la biomasa de malezas entre un $10 \%$ y un $22 \%$ y los peces pueden suprimir las malezas entre un $23 \%$ y un $63 \%$. La integración de patos puede reducir la biomasa de malezas entre el $27 \%$ y el $91 \%$ (Khumairoh, Groot y Lantinga 2012). Además de la supresión de malezas, los peces también pueden regular plagas como las chicharritas (cicadélidos) y enfermedades fúngicas como el tizón de la vaina (causado por Rhizoctonia solani). Los patos tienen una gama aún mayor de especies de presas que los peces, ya que también se alimentan de barrenadores del tallo, chicharritas y otros artrópodos foliares (Xie et ál. 2011).

\section{SISTEMAS AGROBIODIVERSOS}

Los sistemas agrícolas tradicionales sobresalen, entre otros aspectos, por su grado de diversidad vegetal, en forma de policultivos y sistemas agroforestales. Ambos sistemas implican una diversificación que permite mezclar cultivos anuales y arbóreos en distintos esquemas espaciales y temporales. 
Suelen mezclar una leguminosa con un cereal, lo que trae una mayor productividad de la que se obtendría de cada especie por separado, porque las leguminosas fijan nitrógeno, y porque las asociaciones usan los recursos de manera más eficiente $y$, en general, exhiben mayor resistencia a las plagas (Vandermeer 1989).

En los policultivos, las especies crecen muy juntas, permitiendo interacciones beneficiosas y ofrecer una serie de servicios ecosistémicos a los agricultores. La mayor riqueza de especies mejora el contenido en materia orgánica de los suelos, su estructura, su capacidad de retención hídrica y la cubierta, por lo que los suelos se protegen de la erosión y, a la vez, se eliminan malezas, condiciones todas ellas favorables para asegurar la producción. La diversidad de plantas cultivadas también favorece la presencia de artrópodos benéficos y la actividad microbiológica, necesarias para mejorar el reciclaje de nutrientes, la fertilidad de los suelos y la regulación de plagas. Varios estudios demuestran que estos sistemas diversificados exhiben resiliencia frente a los desastres climáticos ya que la presencia de mayor biodiversidad en los campos cultivados aumenta la capacidad adaptativa (Altieri y Nicholls 2004).

Los agricultores de Mesoamérica continúan cultivando maíz en variantes del sistema de milpa -policultivo- en múltiples agroecologías, desde zonas áridas y semiáridas, hasta las tierras altas templadas y las tierras bajas tropicales. Además de intercalar con frijol común y calabaza, el maíz puede cultivarse con habas (Vicia faba), pimientos (Capsicum spp.), Tomate (Solanum lycopersicum), papa (Solanum tuberosum) y amaranto (Amaranthus spp.) e incluso con especies silvestres (quelites) utilizadas como alimento o con fines medicinales (Francis 1986).

En los sistemas agroforestales se intercalan cultivos anuales y perennes o perennes con ganado, a veces con más de cien especies de plantas anuales y perennes y varias especies animales por parcela. Además de proporcionar productos de utilidad (materiales de construcción, leña, herramientas, medicamentos, pienso para el ganado y alimentos), los árboles suelen minimizar la pérdida de nutrientes por lixiviación y erosión, añaden materia orgánica y restauran nutrientes clave, bombeándolos desde las capas inferiores del subsuelo. Los árboles también crean condiciones micro-climáticas que protegen los cultivos y los suelos contra vicisitudes climáticas como tormentas o sequías, que aumentan con el cambio climático (Jose 2009).

En Centroamérica los Sistemas Agroforestales (SAF) de cacao albergan una biodiversidad muy significativa, que incluye 55 familias, 132 géneros y 185 especies de plantas, así como un numero substancial de especies de pájaros (190 especies), murciélagos (36) y varios mamíferos e innumerables invertebrados (Rice y Greenberg 2000). La biodiversidad aumenta en los

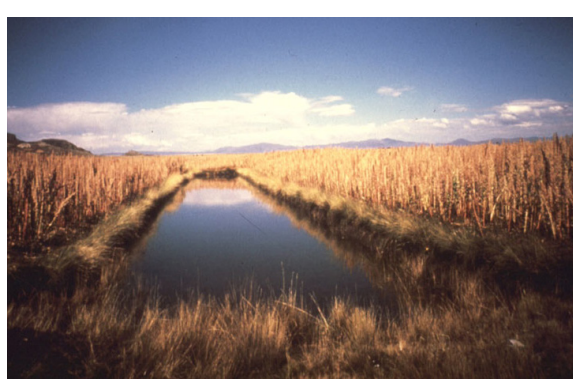

Los waru-warus en el altiplano peruano permiten la producción de cultivos a 3500 msnv en medio de heladas | foto M. C. Altieri 

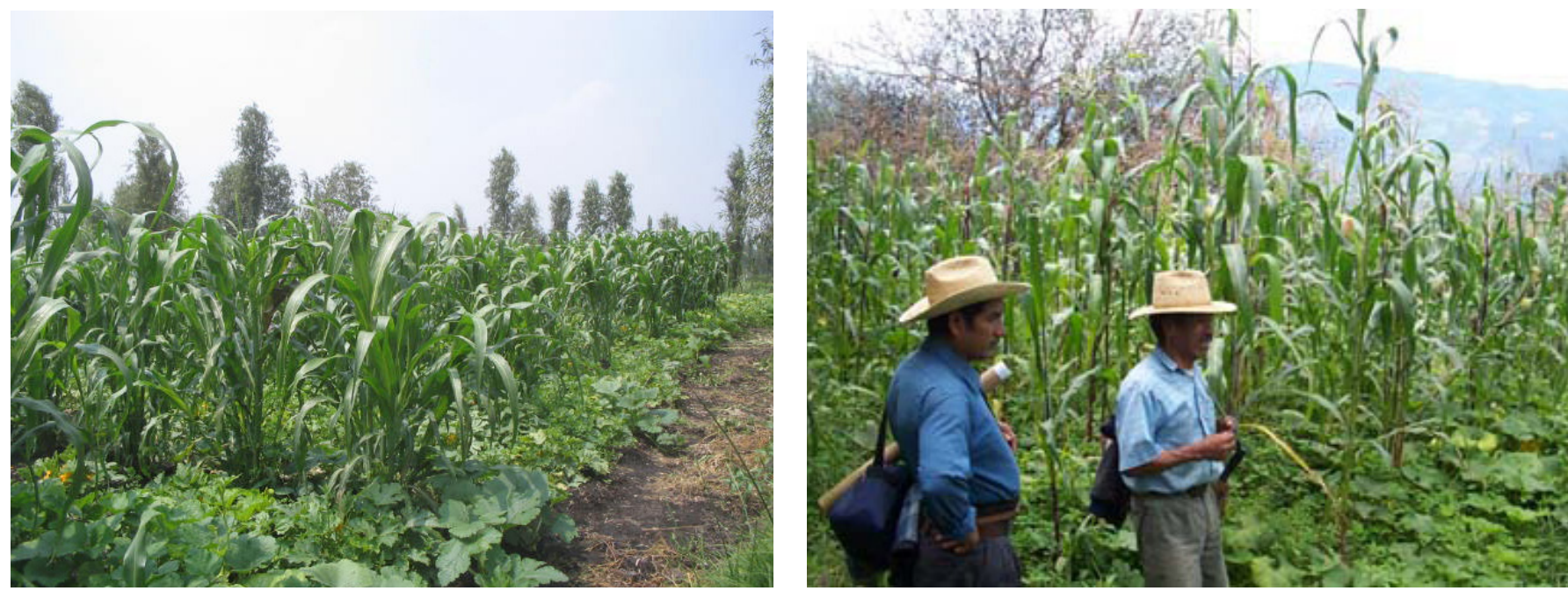

La Milpa, policultivo tradicional mesoamericano (Mexico) | fotos M. A. Altieri (izquierda) y Cesartilan (derecha)

sistemas rústicos multiestrato con un 55-60 \% de sombra y disminuye en los cacaotales con un solo estrato de sombra, máximo dos especies de árboles con una sombra de 35-40 \%. En México, las plantaciones de café bajo sombra sustentan hasta 180 especies de pájaros, entre ellas especies migratorias. Algunas de estas aves desempeñan papeles claves en el control de plagas y la dispersión de semillas (Moguel y Toledo 1999).

En los sistemas silvopastoriles multiestrato (que integran árboles y ganadería), la presencia de árboles leguminosos mejora la producción de pastos y el ciclo de nutrientes, lo que evita añadir fertilizantes químicos nitrogenados a los pastos. Los árboles de raíces profundas ayudan a recuperar nutrientes y agua de las capas profundas del subsuelo e incrementan el secuestro de carbono, tanto bajo tierra como en el follaje. La cubierta arbórea también mejora la oferta alimenticia para el ganado y, a la vez, crea condiciones microclimaticas (sombra, mayor humedad relativa, menor temperatura, etc.). De esta forma, se reduce el estrés de los animales y se mejora su producción y bienestar (Murgueitio et ál. 2011).

\section{DIVERSIDAD GENÉTICA EN LA AGRICULTURA CAMPESINA}

Se estima que los campesinos manejan unas 7000 especies de plantas cultivadas, que incluyen 2,1 millones de variedades, en su mayoría razas locales. Además, se estima que los campesinos mantienen de 50 a 60 mil especies de plantas silvestres emparentadas con los cultivos que se entrecruzan con estos, lo que produce un incremento de la heterogeneidad genética de los sistemas agrícolas. Esta inmensa diversidad genética constituye la base adaptativa de los sistemas agrícolas del ayer, hoy y mañana ya que 


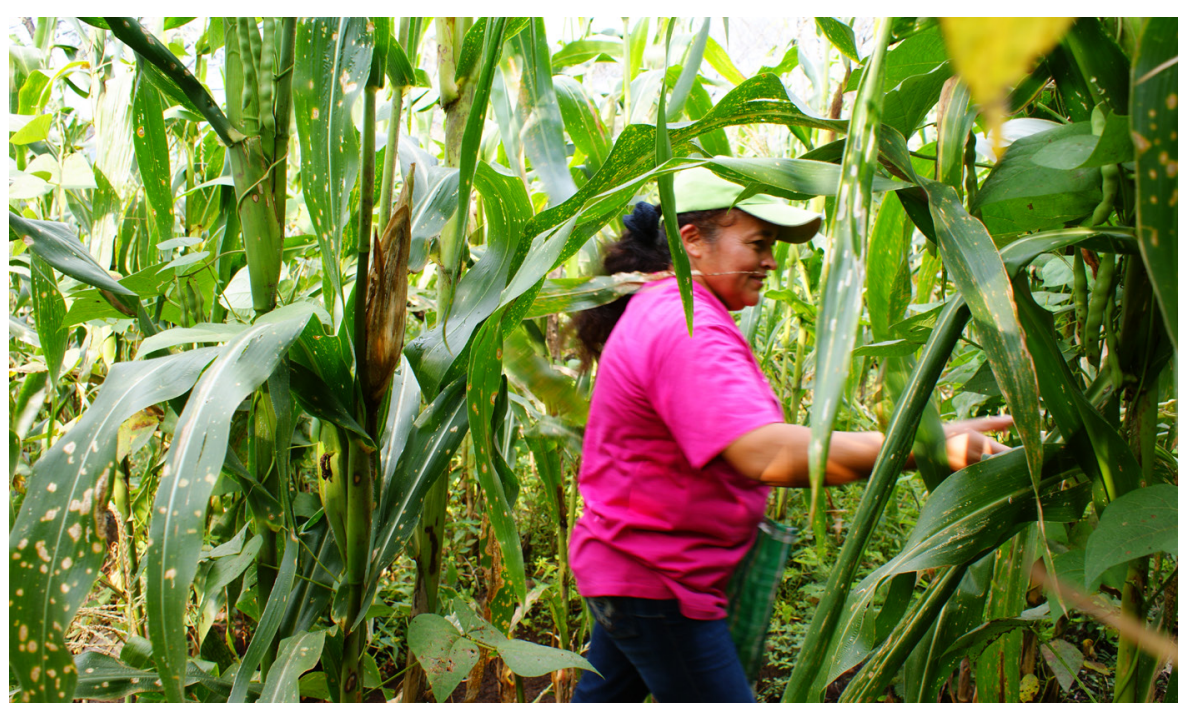

confieren defensas contra la vulnerabilidad e incrementan la seguridad de la cosecha frente a enfermedades, plagas, sequías y otras presiones. Los agricultores obtienen múltiples usos nutricionales y a la vez explotan los varios microclimas y agroecosistemas existentes en cada región, pero que difieren en la calidad del suelo, altitud, pendiente, disponibilidad de agua y otras condiciones (Clawson 1985).

Las mujeres conservan la mayoría de las variedades locales o tradicionales. Las agricultoras aprecian estas variedades por su mejor sabor y propiedades para el almacenamiento, pero también por razones culturales, como su uso simbólico en ceremonias religiosas, regalo en las bodas o recompensa en los trabajos comunitarios. Paradójicamente, tales variedades locales resultan extremadamente importantes para la agricultura industrial, porque contienen una gran cantidad de rasgos necesarios para adaptar los cultivos modernos a las plagas y enfermedades y al clima cambiante.

Gran parte de esta complejidad de los agroecosistemas tradicionales se debe al hecho de que los recursos genéticos de los cultivos suponen más que una colección de alelos y genotipos de cultivos nativos y parientes silvestres. También incluyen interacciones ecológicas como el flujo de genes a través de la polinización cruzada entre las poblaciones y especies de cultivos y la selección y gestión humanas guiadas por sistemas de conocimiento y práctica asociados con la diversidad genética, especialmente taxonomías etnobotánicas y habilidades para seleccionar variedades adaptadas a entornos heterogéneos (Altieri y Merrick 1987). Muchos agricultores "patrocinan" o promueven ciertas malezas o plantas silvestres en o alrededor de sus campos, práctica mediante la cual los agricultores pueden aumentar el flujo de genes entre los cultivos y sus parientes incorporando así varias fuentes de

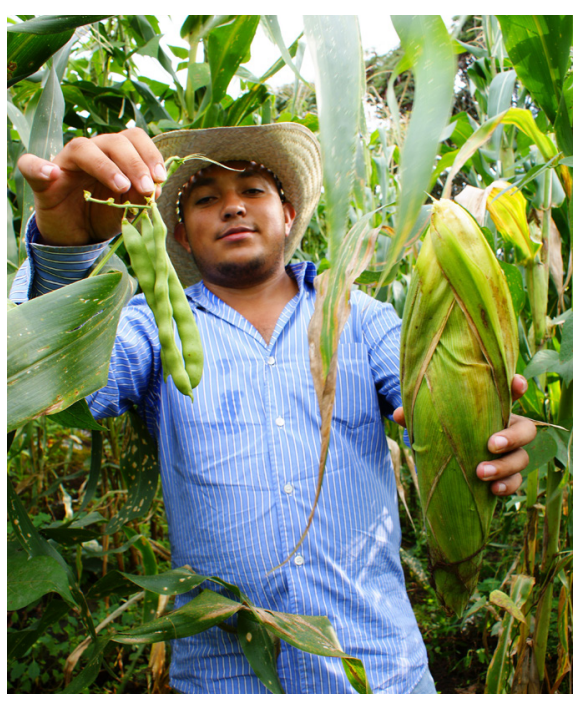

Campesinado de la milpa en Tangamandapio, Michoacán | fotos Feria de Productores 
Factores económicos y socioculturales que determinan el nivel de diversidad genética en

predios campesinos, la cual a su vez proporciona

servicios ecológicos a las familias rurales

resistencia a enfermedades y otras amenazas. Parece claro que la mantención de la diversidad genética en los campos resulta de la interaccion de una serie de factores ecológicos, climáticos, agronómicos y culturales.

En la mayoría de las regiones agrícolas del mundo permanecen agroecosistemas en los cuales los agricultores siembran múltiples variedades de cada cultivo, que brindan diversidad tanto intraespecífica como interespecífica, lo que mejora la seguridad de la cosecha. Por ejemplo, en los Andes los agricultores cultivan hasta 50 variedades de papas en sus campos y, cerca de Ayacucho, los indígenas de Quispillacta mantienen un promedio de 11 especies de cultivos y 74 ecotipos dentro de sus pequeñas parcelas (Brush 2002). De manera similar, en Tailandia e Indonesia los agricultores mantienen una diversidad alta de variedades de arroz en sus campos, adaptadas a una amplia gama de condiciones ambientales. Esta diversidad genética de cultivos tiene un efecto directo en el mantenimiento de los servicios ecosistémicos al proporcionar un mayor número de rasgos funcionales y fomentar interacciones que mantienen la biodiversidad asociada, por encima y por debajo del suelo. Al aumentar la estabilidad del ecosistema a largo plazo frente al estrés biótico y abiótico y a la variabilidad
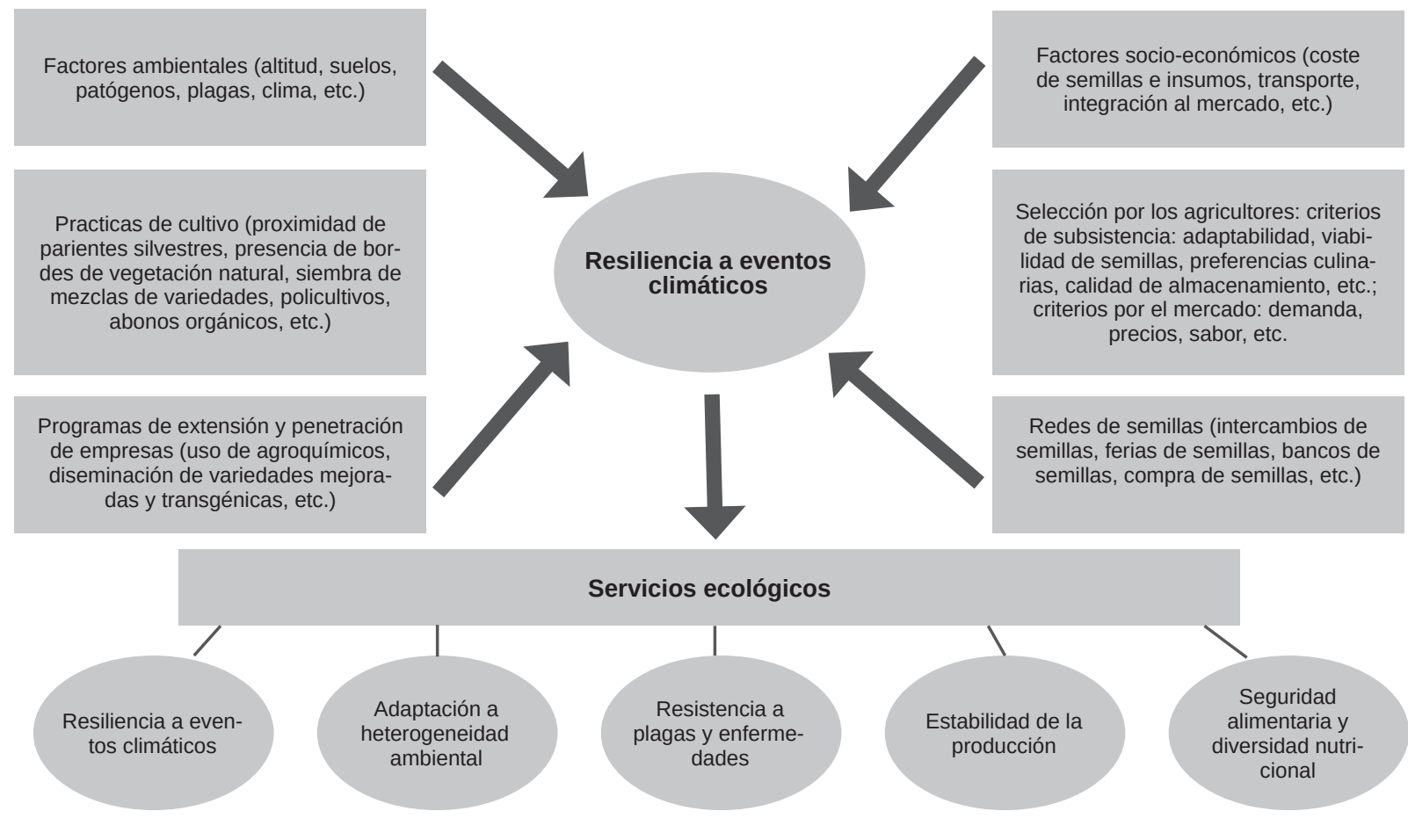
socioeconómica, la diversidad genética promueve el mantenimiento continuo de la biomasa y los servicios ecológicos que esta proporciona.

Como beneficio, la diversidad genética resultante aumenta la resistencia a cepas de enfermedades que atacan determinadas variedades del cultivo. La mezcla de diferentes variedades de cultivos puede retrasar la aparición de enfermedades, reducir la propagación de esporas portadoras de enfermedades y modificar las condiciones ambientales como la humedad, la luz, la temperatura y el movimiento del aire, de modo que sean menos favorables a la propagación de ciertas enfermedades. Un experimento a gran escala en Yunnan, China, donde se plantaron cultivos de arroz genéticamente diversificados, demostró que las variedades de arroz susceptibles a las enfermedades, al ser sembradas en mezclas con variedades resistentes tuvieron un rendimiento $89 \%$ mayor y la infestación de hongos fue $94 \%$ menos severa que cuando se manejaron en cultivos de una sola variedad. El experimento fue tan exitoso que ya no se aplicaron fungicidas después de dos años. Estos resultados ratifican que la diversificación intraespecífica de cultivos constituye un enfoque ecológico sólido para el control de enfermedades (Wolfe 1985).

\section{SISTEMAS AGRÍCOLAS TRADICIONALES COMO MODELOS DE RESILIENCIA}

Al contrario que los monocultivos de la agricultura industrial, muchos sistemas agrícolas tradicionales, que aún persisten en varios países en desarrollo, ofrecen una amplia gama de opciones y diseños de manejo que incrementan la biodiversidad funcional en los campos de cultivo, y, por consiguiente, refuerzan la capacidad de supervivencia de los agroecosistemas (Koohafkan y Altieri 2010; Toledo y Barrera-Bassols 2008). Al tener que lidiar continuamente con fenómenos meteorológicos extremos y la variabilidad climática a través de los siglos, muchos agricultores que viven en entornos hostiles en África, Asia y América Latina han desarrollado y heredado sistemas agrícolas complejos que han permitido a los pequeños agricultores familiares satisfacer sus necesidades de subsistencia en medio de la variabilidad ambiental sin depender de tecnologías agrícolas modernas (Denevan 1995).

Muchas investigadoras e investigadores han señalado que los pueblos indígenas y las comunidades locales, a pesar de su mayor exposición a los riesgos climáticos, están respondiendo activamente a las condiciones climáticas cambiantes y han demostrado su ingeniosidad y adaptación frente al cambio climático. Conservar la diversidad genética y la diversidad de especies en los campos y paisajes aledaños constituyen estrategias que permiten atenuar los riesgos en lugares sometidos a la incertidumbre meteorológica (Altieri et ál. 2013). Con la creación de diversidad temporal y espacial, los campesinos 
tradicionales aumentan la fortaleza de sus agroecosistemas a las fluctuaciones temporales del clima. Una revisión de 172 estudios de caso, así como de informes de proyectos de todo el planeta, demostró que la biodiversidad agrícola tal como la emplean los campesinos tradicionales, contribuye a la supervivencia, mediante el uso de una serie de estrategias a menudo combinadas: la protección y la restauración de los ecosistemas, el uso sostenible de los recursos hídricos y edáficos, la agroforestería, la diversificación de los sistemas agrícolas, diversos ajustes en las prácticas agrícolas y el uso de especies y variedades tolerantes al estrés (Mijatovic et ál. 2013).

Un estudio realizado en las laderas centroamericanas tras el paso del huracán Mitch (1998) sacó a la luz que los campesinos que habían llevado a cabo prácticas de diversificación agroecológica, como los cultivos de cobertura, los policultivos o sistemas agroforestales, sufrieron menos daños por causa del huracán en términos de pérdida de cosechas, erosión edáfica y formación de barrancos y cárcavas, que sus vecinos con monocultivos convencionales. El estudio, liderado por el movimiento Campesino a Campesino (CAC), movilizó un centenar de equipos de campesinos y personal técnico que llevaron a cabo observaciones comparativas de indicadores agroecológicos específicos en 1.804 fincas vecinas, convencionales y agroecológicas. El estudio involucró a 360 comunidades de 24 departamentos en Nicaragua, Honduras y Guatemala. Las parcelas agroecológicas tenían un $20 \%-40 \%$ más de capa vegetal, mayor humedad en el suelo y sufrieron menos erosión y pérdidas económicas que las parcelas vecinas convencionales (HoltGiménez 2002). De modo similar, en el Soconusco, en Chiapas (México), los sistemas de cafetales con altos niveles de complejidad y diversidad vegetal sufrieron menos daños al paso del huracán Stan (2005) que los sistemas de cafetales más simplificados (Philpott et ál. 2008). Cuarenta días después de que el huracán Ike golpease Cuba en 2008, se efectuó una investigación en las provincias de Holguín y Las Tunas, cuya conclusión fue que las fincas agroecológicas diversificadas padecieron pérdidas en torno al $50 \%$, mientras que las de los monocultivos cercanos ascendieron al 90 \%-100 \%. En la misma línea, las fincas manejadas agroecológicamente se recuperaron mucho más rápidamente del daño que las de monocultivo (Rosset et ál. 2011).

En Colombia, los sistemas silvopastorales intensivos (SSPI) suponen una forma sostenible de integración agroecológica basada en la agrosilvicultura con cría de ganado que se alimenta de herbáceas y arbustos plantados en altas concentraciones bajo árboles y palmeras. En 2009, el año más seco en el valle del Cauca, con precipitaciones un $44 \%$ inferiores a la media histórica, estos sistemas obtuvieron un buen rendimiento. A pesar de la reducción de la biomasa de los pastos en un $25 \%$, la producción de alimentos para animales proveniente de árboles y arbustos fue estable durante todo el año y neutralizó los efectos negativos de la sequía a nivel de todo el sis- 
tema. La producción láctea fue la mayor nunca antes registrada, con un sorprendente aumento del $10 \%$ respecto del cuatrienio precedente. Entretanto, en las fincas contiguas con pastizales en monocultivo se produjeron graves pérdidas de peso en los ganados y una alta mortalidad por hambre y sed (Murgueitio et ál. 2011).

Todos los estudios citados hasta aquí subrayan la importancia de aumentar la diversidad y complejidad vegetal en los sistemas agrícolas para reducir la vulnerabilidad a los fenómenos meteorológicos extremos. La literatura indica que los agroecosistemas se vuelven más resilientes si se insertan en una matriz paisajística compleja, con sistemas de cultivo heterogéneos genéticamente y diversificados, manejados con suelos ricos en materia orgánica y técnicas de conservación de agua. Mientras la mayor parte de las investigaciones se centran en la resiliencia ecológica de los agroecosistemas, bien poco se ha escrito aún sobre la resiliencia social de las comunidades rurales que manejan esos agroecosistemas. La capacidad de grupos y comunidades de adaptarse frente a una situación de estrés social, político o medioambiental externo ha de caminar en paralelo a la resiliencia ecológica. Para ser resilientes, las comunidades rurales han de demostrar su capacidad de amortiguar los trastornos con métodos agroecológicos adoptados y difundidos a través de la autoorganización, la reciprocidad y la acción colectiva. Reducir la vulnerabilidad social mediante la extensión y la consolidación de redes sociales, tanto a nivel local como regional, puede contribuir a aumentar la resiliencia de los agroecosistemas. La vulnerabilidad de las comuni-

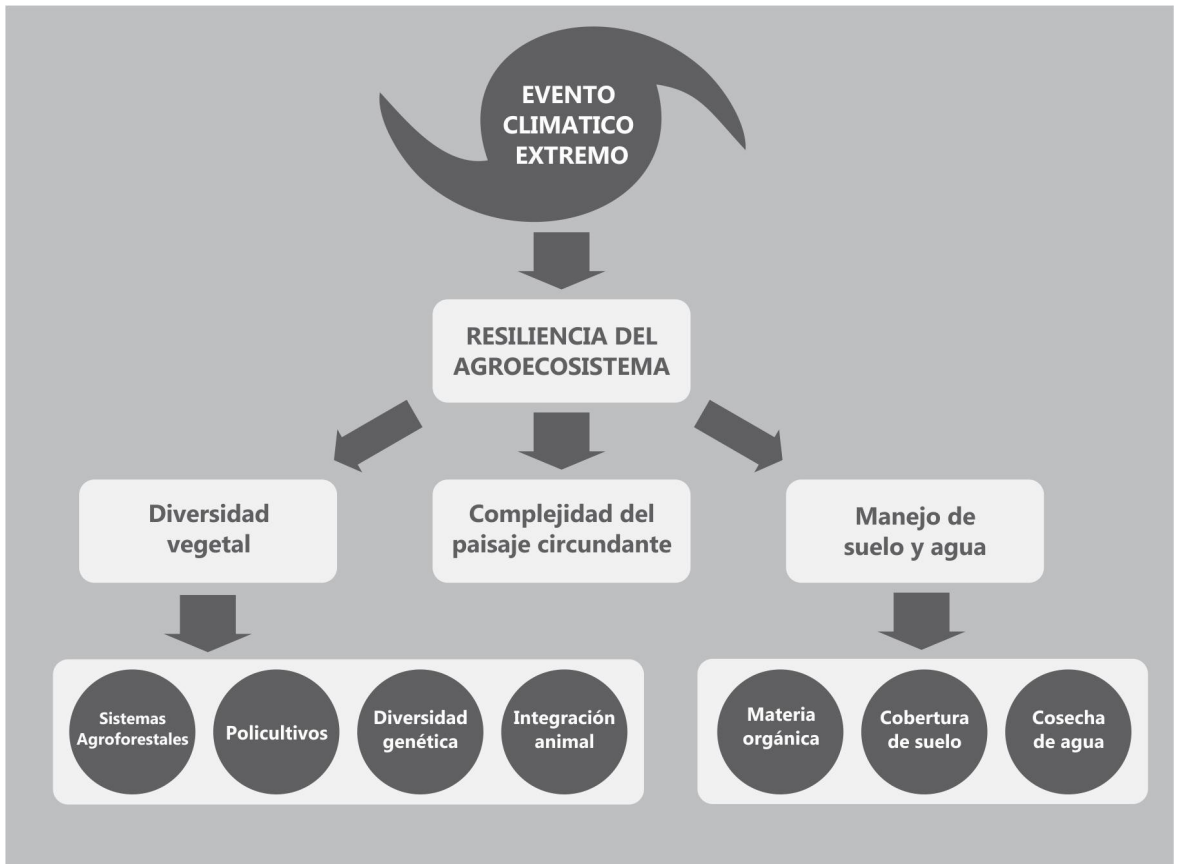


dades agrícolas depende del buen desarrollo de su capital social y natural; el nivel de ese desarrollo determinará si las familias campesinas y sus sistemas de producción seran más o menos vulnerables a los impactos climáticos (Altieri et ál. 2013). La mayoría de las comunidades tradicionales que todavía mantienen sus legados sociales y agroecológicos están dotadas con condiciones para que sus fincas respondan al cambio climático de manera resiliente.

\section{CONCLUSIONES}

En varias regiones rurales del mundo, grupos de seres humanos se han adaptado continuamente a las potencialidades y limitaciones de sus ambientes socioecologicos. Basándose en una riqueza acumulada de conocimientos tradicionales y culturales han modelado paisajes, a los que han dotado de una belleza extraordinaria. Resulta esperanzador observar que bien entrada la segunda década del siglo XXI, millones de campesinos y pequeños agricultores, familiares e indígenas siguen practicando tipologías de agricultura tradicional, que cubren no menos de 10 millones de hectáreas en todo el mundo, y que han demostrado una notable resiliencia a los continuos cambios ambientales y económicos, a la vez que contribuyen sustancialmente a la conservación de la agrobiodiversidad y la seguridad alimentaria a nivel local, regional y nacional.

Independientemente del reconocimiento por la comunidad científica, el conocimiento ancestral que ha guiado la creación de estos agropaisajes sostenibles y resilientes, constituye hoy una base sólida de conocimientos y practicas estratégicas para el desarrollo de innovaciones y tecnologías agrícolas capaces de enfrentar los desafíos que afrontará la agricultura en un futuro cercano. Hay mucho que aprender de los modos de producción autóctonos, ya que estos sistemas tienen una sólida base ecológica, mantienen una valiosa diversidad genética y conducen a la regeneración y preservación de la biodiversidad y los recursos naturales. Los métodos tradicionales parecen particularmente instructivos porque brindan una perspectiva a largo plazo sobre el manejo agrícola exitoso en condiciones de variabilidad climática. Al estudiar estos sistemas, la ciencia y las investigaciones pueden aumentar su conocimiento sobre la dinámica de sistemas complejos, especialmente la relación entre la biodiversidad y la función ecosistémica y también derivar principios prácticos para el diseño de agroecosistemas contemporáneos más sostenibles y resilientes (Altieri 2004).

Muchos de estos sistemas se enfrentan a graves amenazas de diversas fuentes, que incluyen el avance de la agricultura industrial, junto a la penetración de tecnologías foráneas y la globalización que promueve políticas para que los países ricos se deshagan de los excedentes de cereales en países 
de bajos ingresos, política que socava los mercados de los pequeños agricultores. Todas estas amenazas y problemas plantean el riesgo de pérdida de conocimiento ancestral, de una biodiversidad única y agropaisajes excepcionales, que amenaza los medios de subsistencia y la soberanía alimentaria de cientos de comunidades rurales. Lamentablemente, una vez que estos elementos claves y únicos se pierdan, el legado agrícola y los beneficios socioecológicos y culturales asociados, locales y mundiales, también se perderán para siempre.

Por esta razón, la iniciativa de FAO de conservación y gestión adaptativa de los sistemas de patrimonio agrícola de importancia mundial (SIPAM) parece clave para elevar el reconocimiento internacional y el apoyo a la conservación dinámica y la gestión sostenible de dichos sistemas, su agrobiodiversidad y acervo cultural asociados. Parece claro que se necesitan urgentemente políticas para apoyar la conservación dinámica del patrimonio agrícola y salvaguardarlos de los impulsores de cambios externos negativos. Urge asimismo proteger los bienes naturales y culturales de los sitios SIPAM del desarrollo industrial, el cual a menudo extrae mano de obra y causa también distorsión del mercado. Se ha de prestar especial atención a la introducción de variedades modernas e insumos agroquimicos para evitar alterar el equilibrio de los agroecosistemas tradicionales.

Para mejorar la viabilidad económica de estas iniciativas agroecológicas, también se deben desarrollar oportunidades equitativas en los mercados locales y regionales. La experiencia muestra que las políticas publicas pueden apoyar a la agricultura campesina si estas garantizan que las alternativas agroecológicas se adopten de manera amplia y que la producción resultante encuentre salidas garantizadas en los mercados locales. Para conservar e incluso expandir los SIPAM se debe poner especial hincapié en la participación activa de los agricultores en el proceso de innovación tecnológica y difusión a través de faros y modelos Campesino a Campesino que privilegian compartir experiencias y fortalecen la innovación local y las capacidades para la resolución de problemas. Las alianzas de organizaciones rurales con organizaciones de la sociedad civil en zonas urbanas será de suma importancia para crear conciencia entre los consumidores sobre la importancia de mantener y escalonar la agricultura campesina, no solo como fuente de alimentos frescos, sanos y accesibles, sino también como fuente de servicios ecológicos y culturales que benefician a las ciudades. 


\section{BIBLIOGRAFÍA}

- Altieri, M.A. y Merrick, L.C. (1987) In situ conservation of crop genetic resources through maintenance of traditional farming systems. Economic Botany, vol. 41, n. ${ }^{\circ} 1$, pp. 86-96

- Altieri. M.A. (2004) Linking Ecologists and Traditional Farmers in the Search for Sustainable Agriculture. Frontiers in Ecology and the Environment, vol 2, n. ${ }^{\circ} 1$, pp. 35-42

- Altieri, M.A. y Nicholls, C.I. (2004) Biodiversidad y manejo de plagas en agroecosistemas. Barcelona: Icaria

- Altieri, M.A. (2002) Agroecology: the science of natural resource management for poor farmers in marginal environments. Agriculture Ecosystems and Environment, vol. 93, pp. 1-24

- Altieri, M.A. y Toledo, V.M. (2011) The agroecological revolution in Latin America: rescuing nature, ensuring food sovereignty and empowering peasants. The Journal of Pesant Studies, vol. 38, pp. 587-612

- Altieri, M.A., Nicholls, C.I., Henao, A. y Lana, M. (2013) Agroecology and the design of climate change resilient farming systems. Agronomy for Sustainable Development, vol. 35, pp. 869-890

- Beets, W.C. (1982) Multiple cropping and tropical farming systems. Boulder: Westview Press

- Browder, J.O. (1989) Fragile Lands in Latin America: Strategies for Sustainable Development. Boulder: Westview Press

- Brush, S.B. (2000) Genes in the field: on farm conservation of crop diversity. Boca Raton, FL: Lewis Publishers

- Clawson, D.L. (1985) Harvest Security and Intraspecific Diversity in Traditional Tropical Agriculture. Economic Botany, vol. 39, pp. 56-67

- Denevan, D.M. (1995) Prehistoric Agricultural Methods as Models for Sustainability, Advanced Plant Pathology, vol. 11, pp. 21-43

- Dewalt, B.R. (1994) Using indigenous knowledge to improve agriculture and natural resource management. Human Organization, vol. 5, pp. 23-131

- Francis, C.A. (1986) Multiple Cropping Systems. New York: Mac- Millan

- Hanks, L. (1992) Rice and Man: Agricultural Ecology in Southeast Asia. Honolulu: University of Hawaii Press

- Holt-Giménez, E. (2002) Measuring farmers agroecological resistance after Hurricane Mitch in Nicaragua: a case study in participatory, sustainable land management impact monitoring. Agric. Ecosyst. Environ., vol. 93, pp. 87-105.

- Koohafkan, P. y Altieri, M.A. (2017) Forgotten agricultural heritage: reconnecting food systems and sustainable development. London, Routledge

- Koohafkan, P. y Dela Cruz, M.J. (2011) Conservation and adaptive management of Globally Important Agricultural Heritage Systems (GIAHS). Journal of Resources and Ecology, vol. 2 n. ${ }^{\circ}$, pp. $22-28$

- Khumairoh, U., Groot, J.C.J. y Lantinga, E.A. (2012) Complex agro-ecosystems for food security in a changing climate. Ecol. Evol., vol. 2, n. ${ }^{\circ} 7$, pp. 1696-1704

- Jose, S. (2009) Agroforestry for ecosystem services and environmental benefits: an overview. Agroforestry Systems, vol. 76 , pp. 1-10

- Mijatovic, D., Van Oudenhovenb, F., Eyzaguirreb, P. y Hodgkins, T. (2013) The role of agricultural biodiversity in strengthening resilience to climate change: towards an analytical framework. Int. J. Agric. Sustain., vol. 11, n. ${ }^{\circ} 2$, pp. 23-31

- Moguel, P. y Toledo, V.M. (1999) Biodiversity Conservation in Traditional Coffee Systems of Mexico. Conservation Biology, vol. 13, n. ${ }^{\circ}$ 1, pp. 11-21

- Murgueitio, E., Calle, Z., Uribe, F., Calle, A. y Solorio, B. (2011) Native trees and shrubs for the productive rehabilitation of tropical cattle ranching lands. Forestry, Ecology and Management, vol. 261, pp. 1654-1663

- Ortega, E. (1986) Peasant Agriculture in Latin America. Santiago: Joint ECLAC/ FAO Agriculture Division

- Philpott, S.M, Lin, B.B, Jha, S. y Brines S.J. (2009) A multiscale assessment of hurricane impacts on agricultural landscapes based on land use and topographic features. Agric. Ecosyst. Environ., vol. 128, pp. 12-20

- Reij, C., Scoones, I. y Toulmin, C. (1996) Sustaining the soil: indigenous soil and water conservation in Africa. London: Earthscan

- Rice, R.A. y Greenberg, R. (2000) Cacao cultivation and the conservation of biological diversity. Ambio, vol. 29, pp. 167-173

- Richards, P. (1985) Indigenous Agricultural Revolution. Boulder: Westview Press

- Rosset, P.M., Machín-Sosa, B., Roque-Jaime, A.M. y Ávila-Lozano, D.R. (2011) The Campesino-to-Campesino agroecology movement of ANAP in Cuba. J. Peasant Stud., vol. 38, n. ${ }^{\circ} 1$, pp. 161-191

- Toledo, V.M., Carabias, J., Mapes, C. y Toledo, C. (1985) Ecologia y Autosuficiencia Alimentaria. Mexico: Siglo Veintiuno

- Toledo, V.M. y Barrera-Bassols, N. (2008) La Memoria Biocultural: la importancia ecológica de las sabidurías 
tradicionales. Barcelona: ICARIA

- Vandermeer, J. (1992) The ecology of intercropping. New York, NY: Cambridge University Press

- Wilken, G.C. (1987) Good Farmers: traditional agricultural resource management in Mexico and Guatemala. Berkeley: University of California Press

- Wolfe, M.S. (1985) The current status and prospects of multiline cultivars and variety mixtures for disease resistance. Annu. Rev. Phytopathol, vol. 23, pp. 251-273

- Xie, J., Hu, L., Tang, J., Wu, X., Li, N., Yuan, Y. y Chen, X. (2011) Ecological mechanisms underlying the sustainability of the agricultural heritage rice-fish coculture system. Proceedings of the National Academy of Sciences, vol. 108, n. ${ }^{0}$ 50, pp. E1381-E1387 